\title{
Integration of Contact Size Dependence and Thermal Activation in Atomic Friction
}

\author{
Jiangnan Zhang, , Jin Haeng Lee, Jun Lou, a,* Yanfei Gao ${ }^{\mathrm{c}, \mathrm{d}, *}$ \\ ${ }^{a}$ Department of Materials Science and NanoEngineering, Rice University, Houston, TX 77005, USA \\ ${ }^{\mathrm{b}}$ Research Reactor Mechanical Structure Design Division, Korea Atomic Energy Research Institute, \\ Daejeon 305-353, Republic of Korea \\ ${ }^{c}$ Department of Materials Science and Engineering, University of Tennessee, Knoxville, TN 37996, USA \\ ${ }^{\mathrm{d}}$ Materials Science and Technology Division, Oak Ridge National Laboratory, Oak Ridge, TN 37831, USA
}

Nano-asperity friction force has been found to depend on both the sliding velocity and the contact size. The former is a consequence of stress-assisted, thermally activated process, which is often explained by the one-degree-of-freedom Tomlinson model that assumes a virtual point contact sliding over a periodic tip-surface interaction potential. However, this model cannot provide an accurate description of the rate-determining process when coupled with the contact size dependence. In this synergistic experimental/modeling study, atomic friction measurements were conducted on cleaved mica and HOPG surfaces with varying normal force and sliding velocity, and the spatially-nonuniform saddle-point configurations were calculated from a Peierls framework. The agreement between experiments and theory suggest that the detailed characteristics of the interface slip field govern the atomic friction.

Keywords: atomic friction, thermally activated process

\footnotetext{
*Corresponding authors: jlou@ rice.edu; ygao7@utk.edu 
One critical question in the study of mechanically interacting rough surfaces is how single asperities deform in response to the normal and shear forces. ${ }^{1-5}$ In the past several decades, the development of friction force microscopy (FFM) has enabled the quantitative measurement of friction behavior for nanoscale asperities with the contact sizes lying below $\sim 100 \mathrm{~nm}$. The first noteworthy observation is the serrated force-displacement curves with stick-slip periodicity matching the substrate lattice constants. The Prandtl-Tomlinson model is often used to explain this observation, in which a point contact is sliding over a periodic potential that represents the tip-substrate interaction ${ }^{4,5}$ The point contact is connected to the loading apparatus (i.e., the cantilever in the friction force microscopy), and the low spring constant and high magnitude of the corrugated potential lead to the stick-slip behavior. While the model has been successfully applied in atomic friction, one obvious drawback is that all the interface atoms in contact are "forced" to move simultaneously, i.e., only one degree of freedom is allowed. We now address the consequences of this drawback in several recent experiments. Socoliuc et al. ${ }^{6}$ found that the stick-slip behavior may disappear when the normal contact is reduced. Their interpretation based on Tomlinson model is the reduction of the corrugated potential magnitude (as the decrease of the applied normal force). The required reduction was found to be one or two orders of magnitude. In contrast, we view that the friction process involves an inhomogeneous slip of the interface atoms, and the boundary between atoms with slip displacement differing by one lattice constant is a dislocation. When the contact size is larger than the dislocation core size (i.e., the distance that slip field varies rapidly by one Burgers vector in a Peierls dislocation viewpoint), the friction behavior is achieved by dislocation nucleation and motion which result into a stress drop on the force-displacement curve. The disappearance of the stickslip behavior corresponds to a small contact size that is comparable to or smaller than the dislocation core size. More importantly, this Peierls perspective does not invoke the change of the corrugated potential magnitude.

Another drawback with the Tomlinson model is related to the thermally activated friction. The atomic friction force often exhibits a logarithmic dependence on the sliding velocity. ${ }^{8,9}$ Gnecco et al. ${ }^{8}$ tested the frictional behavior of $\mathrm{NaCl}$ (100) surface with normal force on the order of nano-Netwons and with the sliding velocity, $V$, ranging from $5 \mathrm{~nm} / \mathrm{s}$ to $1 \mu \mathrm{m} / \mathrm{s}$. The friction force is found to be proportional to $\ln V$. A subsequent work by Riedo et al. ${ }^{9}$ for cleaved mica surface demonstrated further that there is an asymptotic value of the friction force at very large $V$, and the variation of normal force (from 4 to $12 \mathrm{nN}$ ) does not change the $\ln V$ dependence. Also if the surrounding temperature decreases, the friction force will increase. ${ }^{10-12}$ For instance, Jansen et al. ${ }^{12}$ measured the friction force on a highly ordered pyrolytic graphite (HOPG) surface and showed the temperature dependence of friction force in the range of 100$300 \mathrm{~K}$. All these observations suggest that the friction is a stress-assisted, thermally activated process. When tested at low velocity and high temperature, even if the applied force is lower than the athermal 
friction force, the atoms in contact may have a longer time (in the case of low sliding velocity) or a higher kinetic energy (in the case of high temperature), and thus a higher probability, to overcome the activation energy barrier. In spite of the wide acceptance of this view, we call into questions the determination of the activation energy by Tomlinson model. That is, in both Gnecco et al. ${ }^{8}$ and Riedo et al. ${ }^{9}$ that use the onedegree-of-freedom Tomlinson model, the corresponding saddle-point configuration for the activation energy calculation is apparently a homogeneous slip, which cannot treat contact size dependence as explained in the preceding paragraph.

In view of the restricted homogeneous motion of all interface atoms in the Tomlinson model, we argue that the fortuitous success of this model is the nanometer-sized contact in these experiments, The Peierls dislocation model $^{7}$ will degenerate into the one-degree-of-freedom Tomlinson model at small contacts, but will deviate from it when the contact size is larger than the dislocation core size. The hypothesis to be tested is that if the activated energy can be accurately calculated from an inhomogeneous slip of the interface atoms without the need of changing the magnitude of the corrugated potential, how will the results be compared to atomic friction measurements with a wide change of contact size and sliding velocity? Next we will present both theoretical calculations and experimental measurements.

The FFM experiments were conducted in an Atomic Force Microscopy (Agilent PicoPlus) in an atmosphere control chamber, where a small amount of nitrogen gas was continuously flown into this chamber. The cantilever had a monolithic silicon probe with a nominal radius of $10 \mathrm{~nm}$. The normal force constant was in situ calibrated using a standard pre-calibrated cantilever with known bending stiffness. A simple and reliable way for friction force calibration with high accuracy and effectiveness was performed in situ using the recently developed levitation method. ${ }^{13-15}$ The resulting normal and lateral stiffnesses were $k_{\text {normal }}^{\text {cantilever }}=0.096 \mathrm{~N} / \mathrm{m}$ and $k_{\text {lateral }}^{\text {cantilev }}=1.14 \mathrm{~N} / \mathrm{m}$, respectively. In our first set of experiments, freshly cleaved and atomically smooth mica surfaces were used at room temperature and low humidity $(<4 \%)$. The second set was conducted on freshly cleaved HOPG at room temperature and low humidity $(2.7 \pm 0.2 \%)$. Different scan dimensions were employed in order to obtain various scanning velocities in Fig. 1. Each data point in Fig. 1 corresponds to one friction measurement, from which the friction force is calculated as the half of the stick-slip peak force during forward scanning subtracted by the peak force during backward scanning.

Our measurements in Fig. 1 have the same characteristics as the previous measurements. ${ }^{8,9}$ In a wide range of sliding velocity, the friction force $F_{L}$ is proportional to $\ln V$. Eventually at sufficiently high $V$, the friction force reaches a constant, which is the athermal friction force $F_{L}^{a t h e r m a l}$. With respect to the increase of the normal force, the friction force increases accordingly, and the $\ln V$ dependence remains the same for all the five loads and for the two materials studied in our FFM measurements. Note 
that $F_{N}$ can be negative because of the adhesion between the cantilever tip and the substrate surface. One can study the relationship between asymptotic values, $F_{L}^{\text {athermal }}$, and the normal force $F_{N}$. If the contact size can be calculated from the Johnson-Kendall-Roberts (JKR) fitting with the knowledge of $F_{N}$, we can find the shear strength of the frictional interface, or called the friction stress, by $F_{L}^{a t h e r m a l} / \pi a^{2}$. Such a procedure has been routinely conducted in FFM investigations. Here our focus is rather placed on information that can be extracted from the dependence on velocity and $F_{N}$. To this end, presented next is the theoretical calculations of the activation energy.

In our recently developed Peierls framework, ${ }^{7}$ the total potential energy is given by

$$
\Pi=\Pi_{0}+\int_{S} \Gamma\left(\Delta_{\alpha}\right) d S+\frac{1}{2} \int_{S} n_{i} \sigma_{i \alpha}^{\text {self }} \Delta_{\alpha} d S-\int_{S} n_{i} \sigma_{i a}^{\text {applied }} \Delta_{\alpha} d S
$$

where $\Delta_{\alpha}=\left(\Delta_{x}, \Delta_{y}\right)$ is the relative in-plane slip between atoms on the two contacting surfaces, and the normal of the interface $S$ is $n_{i}$. On the right hand side of Eq. (1), the first term is the potential energy of the contact problem without any interface slip. The second term represents the excessive interface energy, $\Gamma\left(\Delta_{\alpha}\right)$, or called the $\gamma$ surface, which is caused by the introduction of the interface slip, similar to the tip-substrate interaction potential in Tomlinson model. Note that Tomlinson model implicitly assume $\Delta_{\alpha}$ being constants. Here we use a simple sinusoidal representation, $\partial \Gamma / \partial \Delta_{\alpha}=\tau_{\max } \sin \left(2 \pi \Delta_{\alpha} / b\right)$, with $\tau_{\max }$ being the theoretical shear strength of the interface and $b$ being the Burgers vector. The third term is the elastic strain energy due to interface slip with $\sigma_{i j}^{\text {self }}$ being the self-stress arising from the nonuniform interface slip, and the last term is the interaction energy between the outside applied field, $\sigma_{i j}^{a p p l i e d}$, and the interface slip. The nonuniform interface slip is so developed that a diffused-core dislocation may initiate from the contact edge, propagate inward, and annihilate at the center, as depicted in the top row in Fig. 2(a). If the dislocation core size (that scales roughly as $0.2 \mu \mathrm{b} / \tau_{\max }$ with shear modulus $\mu$ ) is comparable to or smaller than the contact size, the entire interface slips uniformly as in the Tomlinson scenario.

The energy functional in Eq. (1) permits a quantitative calculation of the activation energy. When the applied shear force is lower than the athermal friction force, i.e., $F_{L}<F_{L}^{\text {athermal }}$, there will be two stationary solutions that satisfy $\delta \Pi\left\{\Delta_{\alpha}(x, y)\right\}=0$, where $\delta(.$.$) is the functional differential operator.$ One solution is metastable (minimum energy) and the other is the saddle point configuration, and the 
activation energy is given by $\Delta \Pi=\Pi\left(\Delta_{\alpha}^{\text {saddle }}\right)-\Pi\left(\Delta_{\alpha}^{\text {metastable }}\right)$. Only asymptotic solutions were given in our previous work, ${ }^{7}$ from which the following representation of the activation energy is suggested,

$$
\frac{\Delta \Pi}{\mu b^{3}}=\beta^{*}\left(1-\frac{F_{L}}{F_{L}^{\text {athermal }}}\right)^{3 / 2} \text {. }
$$

For a very large contact size $\left(a>>\mu b / \tau_{\max }\right)$, the prefactor $\beta^{*}$ is roughly a constant, being about 10 from the study of dislocation nucleation from a crack tip. ${ }^{16}$ For small contacts, $\beta^{*}$ should increase monotonically with respect to $a$, but no analytical treatment is available. A finite element method has been developed to find the stationary points of the energy functional. ${ }^{17}$ The minimum energy solution can be easily obtained by applying the shear force from zero to $F_{L}$; this is the equilibrium pathway. The saddle point configuration is found from an initial trial state, ${ }^{16-18}$ which resembles a half dislocation loop nucleated from the contact edge as shown in the bottom row in Fig. 2(a). Starting from such a trial solution, we use the Newton-Raphson method to find out the saddle point configuration that reaches $\delta \Pi=0$. Numerical results in Fig. 2(b) illustrate examples of the metastable and saddle point configurations, i.e., $\Delta_{x}(x, y)$, which correspond to the two red stars on Fig. 2(c). Figure 2(c) gives the normalized activation energy, $(1-v) \Delta \Pi / \mu b^{3}$, as a function of $F_{L} / \pi a^{2} \tau_{\max }$ with Poisson's ratio $v$, $\tau_{\max } / \mu=0.1$, and several representative values of $a / b$. When $F_{L}$ is much less than $F_{L}^{\max }$, the saddle point configuration does exhibit the shape of the half dislocation loop, as shown by the example of $F_{L} / \pi a^{2} \tau_{\max }=0.7$ in Fig. 2(b). But when $F_{L}$ approaches $F_{L}^{\max }$, the saddle point configuration assumes a similar shape as the metastable solution, as shown by the example of $F_{L} / \pi a^{2} \tau_{\max }=0.89$ in Fig. 2(b). Results in Fig. 2(c) differ slightly from the power-law form in Eq. (2). Nevertheless, as the overall trend, with the increase of the contact size, the athermal friction force decreases (i.e, the ratio of $F_{L}^{\text {athermal }} / \pi a^{2} \tau_{\max }$ decreases with respect to the increase of $\left.a / b\right)$, and the slope of $\Delta \Pi \sim F_{L}$ curves becomes significantly large.

Next we apply the activation energy in Eq. (2) to study the velocity dependence in Fig. 1. The use of an Arrhenius law gives the transition rate $\dot{n}$ (i.e., the number of transitions per unit time per unit area on the interface), $\dot{n}=\dot{n}_{0} \exp \left[-\Delta \Pi\left(F_{L}\right) / k_{B} T\right]$, where $\dot{n}_{0}$ is an attempt frequency per unit interface area, $k_{B}$ is the Boltzmann constant, and $T$ is the absolute temperature. In the transition state theory, the 
cumulative transition probability, $f$, obeys $\dot{f}=\pi a^{2} \dot{n}(1-f) \cdot{ }^{8,9,16,18}$ The measured friction force will have a Gaussian distribution, and the mean value corresponds to $d^{2} f / d F_{L}^{2}=0$, given by

$$
\frac{\mu b^{3} \beta^{*}}{k_{B} T}\left(1-\frac{F_{L}}{F_{L}^{\text {athermal }}}\right)^{3 / 2}+\frac{1}{2} \ln \left(1-\frac{F_{L}}{F_{L}^{\text {athermal }}}\right)=\ln \frac{V_{0}}{V}
$$

with $V_{0}=\frac{2 \pi \dot{n}_{0} k_{B} T a^{2} F_{L}^{a t h e r m a l ~}}{3 k_{\text {eff }} \beta^{*} \mu b^{3}}$. Note that $\dot{n}_{0}$ may also depend on the contact size. Consequently, besides the contact size dependence of friction stress $\left(F_{L}^{\text {athermal }} / \pi a^{2}\right)$, the thermally activated friction has an additional area dependence from the activation energy $\Delta \Pi\left(F_{L} ; a\right)$ and the availability of attempt sites. In the above derivation, the effective stiffness is a composite of the cantilever stiffness $k_{\text {lateral }}^{\text {cantiler }}$ and the shear stiffness of the contact, given by $k_{\text {eff }}=\left[1 / k_{\text {lateral }}^{\text {cantilever }}+(2-v) / 8 a \mu\right]^{-1} \cdot{ }^{19}$ The cantilever and contact stiffnesses are comparable in our experiments.

When fitting the model in Eq. (3) to the measurements in Fig. 1, three fitting parameters are needed, including $F_{L}^{a t h e r m a l}$ that can be read from the plateau value at high velocity, $\beta^{*}$ that determines the slope of these curves, and $V_{0}$ that only translates the fitting curves horizontally. Since our objective is to test the contact size dependence of $\Delta \Pi\left(F_{L} ; a\right)$ as given in Eq. (2) and in Fig. 2(c), we focus on the relationship between $\beta^{*}$ and $F_{N}$ (or $a$ ). As shown in Fig. 3(a), the fitted values of $\beta^{*} \mu b^{3} / k_{B} T$ are almost the same for both mica and HOPG surfaces, and $\beta^{*}$ increases monotonically with respect to $F_{N}$. The determination of $a$ requires a nor mal contact analysis. We find that for all our experiments with normal forces given in Fig. 1, the contact area and the critical pull-off forces can be expressed in the JKR solution for adhesive normal contact, ${ }^{1}$ in which the elastic deformation caused by adhesive forces is large compared to their range of action. The detailed JKR fitting procedure can be found elsewhere, ${ }^{14,15}$ and the normal force levels in Fig. 1 give rise to contact sizes varying in about 3-6 nm for mica and in about 4-7 $\mathrm{nm}$ for HOPG, as given in Fig. 3(c). In these calculations, the Young's modulus $E$ and Poisson's ratio $v$ are taken as $70 \mathrm{GPa}$ and 0.17 respectively for silicon oxide, $60 \mathrm{GPa}$ and 0.25 for mica, and $3.5 \mathrm{GPa}$ and 0.25 for HOPG. ${ }^{19}$

Combining $\beta^{*}$ in Fig. 3(a) and $a$ in Fig. 3(c), we now examine how the dependence of $\beta^{*}(a)$ compares to the theoretical calculations. The upper bound of $\beta^{*}(a)$ at large $a$ corresponds to the dislocation nucleation from a crack tip, being about 10 in the analysis by Rice and Beltz. ${ }^{16}$ The lower 
bound of $\beta^{*}(a)$ at small $a$ corresponds to just one atom in contact, being roughly the ratio of interface theoretical strength to the shear modulus, $\tau_{\max } / \mu$, which is about $1 / 10$ for most materials. ${ }^{7}$ The transition of these two limits is governed by the comparison of dislocation core size and contact size, i.e., $0.2 \mu \mathrm{b} / \tau_{\max }$ versus $a$. For the silicon tip scanning on the mica surface, we need to use the composite shear modulus, which is about $10 \mathrm{GPa}$. The Burgers vector is about $0.2 \mathrm{~nm}$. The theoretical strength can be estimated from $F_{L}^{\text {athermal }}$ and $a$. For example, at $F_{N}=-5 \mathrm{nN}, F_{L}^{a \text { thermal }}$ is about $16 \mathrm{nN}$ and $a$ is about $3.2 \mathrm{~nm}$ for mica data in Fig. 1(a). The friction stress is determined to be about $0.5 \mathrm{GPa}$. Thus the dislocation core size is $0.2 \mu \mathrm{b} / \tau_{\max } \approx 0.8 \mathrm{~nm}$. Compared to the contact size in Fig. 3(c), we see that not only the calculations in Fig. 2(c) give reasonable values of $a / b$, but also the interface inhomogeneous slip behavior lies in between the Tomlinson limit and the limit that corresponds to the nucleation of dislocation from a crack tip. Consequently, it is anticipated that we will see a variation of $\beta^{*}$ in the range of 0.1-10 from these two limits. We also note $\mu b^{3} / k_{B} T \approx 20$ when using $\mu=10 \mathrm{GPa}, b=0.2 \mathrm{~nm}$, and $k_{B} T=4.1 \times 10^{-21} \mathrm{~J}$ at room temperature. Thus results in Fig. 3(a) give a variation of $\beta^{*}$ in the range of $0.3-0.5$, which agree perfectly with our theoretical discussion in the above. This agreement verifies the contact size dependence of the activation energy through $\beta^{*}(a)$.

As a consistency check, we note that the product of the fitted values of $\beta^{*} \mu b^{3} / k_{B} T$ and $V_{0}$ should agree with $V_{0} \beta^{*} \mu b^{3} / k_{B} T=\left(2 \pi \dot{n}_{0} / 3 k_{e f f}\right) a^{2} F_{L}^{a t h e r m a l}$. Combining Figs. 3(a) and 3(b), it is found that $V_{0} \beta^{*} \mu b^{3} / k_{B} T$ varies by a factor of about $4-5$, which agree perfectly with the similar increase of $a^{2} F_{L}^{a \text { thermal }}$, since $F_{L}^{a \text { athermal }}$ increases by a factor of 2-3 and $a$ by a factor of about 1.5 .

In summary, a synergistic modeling/experimental study has been performed for the integration of contact size dependence and velocity dependence (as an outcome of thermal activation) in atomic friction. Previous works using the Tomlinson model need to assume a n ormal-force dependence of the tipsubstrate corrugated potential, while our Peierls-type model has not this restriction and thus determines the activation energy properly from spatially nonuniform saddle-point configurations. Activation energy obtained by fitting to the measured $F_{L} \sim \ln V$ curves agree nicely with the numerical calculations, both of which confirm the size dependence in $\Delta \Pi\left(F_{L} ; a\right)$.

\section{Acknowledgements}


This work was supported by the US Department of Energy, Office of Basic Energy Sciences under contract DE-FG02-10ER4677 (JNZ and JL), and by the National Science Foundation under grant numbers CMMI 0900027 (JHL and YFG) and CMMI 1300223 (YFG). 


\section{References}

1. R.W. Carpick and M. Salmeron, Chem. Rev. 97, 1163 (1997).

2. Y.F. Gao and A.F. Bower, Proc. R. Soc. A 462, 319 (2006).

3. Q. Li and K.-S. Kim, Proc. R. Soc. A 464, 1319 (2008).

4. H. Hölscher, A. Schirmeisen, and U.D. Schwarz, Phil. Trans. R. Soc. A 366, 1383 (2008).

5. I. Szlufarska, M. Chandross, and R.W. Carpick, J. Phys. D: Appl. Phys. 41, 123001 (2008).

6. A. Socoliuc, R. Bennewitz, E. Gnecco, and E. Meyer, Phys. Rev. Lett. 92, 134301 (2004).

7. Y.F. Gao, J. Mech. Phys. Solids 58, 2023 (2010).

8. E. Gnecco, R. Bennewitz, T. Gyalog, Ch. Loppacher, M. Bammerlin, E. Meyer, and H.-J. Güntherodt, Phys. Rev. Lett. 84, 1172 (2000).

9. E. Riedo, E. Gnecco, R. Bennewitz, E. Meyer, and H. Brune, Phys. Rev. Lett. 91, 084502 (2003).

10. X. Zhao, S.R. Phillpot, W.G. Sawyer, S.B. Sinnott, and S.S. Perry, Phys. Rev. Lett. 102, 186102 (2009).

11. A. Schirmeisen, L. Jansen, H. Hölscher, and H. Fuchs, Appl. Phys. Lett. 88, 123108 (2006).

12. L. Jansen, H. Hölscher, H. Fuchs, and A. Schirmeisen, Phys. Rev. Lett. 104, 256101 (2010).

13. Q. Li, A. Rydberg, and K.-S. Kim, Rev. Sci. Instr. 77, 065105 (2006).

14. J. Lou and K.-S. Kim, Mater. Sci. Eng. A 483-484, 664 (2008).

15. J. Zhang, W. Lu, J.M. Tour, and J. Lou, Appl. Phys. Lett. 101, 123104 (2012).

16. J.R. Rice and G.E. Beltz, J. Mech. Phys. Solids 42, 333 (1994).

17. J.H. Lee and Y.F. Gao, Int. J. Solids Struct. 48, 1180 (2011).

18. T.L. Li, Y.F. Gao, H. Bei, and E.P. George, J. Mech. Phys. Solids 59, 1147 (2011).

19. Y.F. Gao, H.T. Xu, W.C. Oliver, and G.M. Pharr, J. Mech. Phys. Solids 56, 402 (2008).

20. M. Enachescu, D. Schleef, D.F. Ogletree, and M. Salmeron, Phys. Rev. B 60, 16913 (1999). 


\section{Figure Captions}

Figure 1 Friction force (extracted as the half value of the bounds of the stick-slip curves during forward and background sliding tests) increases as a function of sliding velocity. Solid lines are fitting results from theoretical model in Eq. (3). FFM experiments were conducted on (a) mica and (b) HOPG surfaces.

Figure 2 (a) Schematic illustration of the sequence of inhomogeneous slip on the interface in one stick-slip period. Dislocated and un-dislocated areas are marked white and hashed, respectively. The boundary is the dislocation denoted by the dashed curve. Top row is for the athermal process at zero $\mathrm{K}$, and the bottom row shows the thermally activated slip initiated from one side of the contract zone at finite temperature. (b) Metastable and saddle point configurations for $a / b=10$ and $\tau_{\max } / \mu=0.1$ at two representative applied lateral force, as marked by red stars in (c). (c) Activation energy as a function of the applied friction force. With the increase of contact size, the activation energy increases more rapidly when the applied shear force is lower than the athermal limit.

Figure 3 (a) Fitted prefactor of the activation energy in Eq. (2), and (b) fitted characteristic velocity in Eq. (3) from experimental data in Fig. 1. (c) The relationship between $F_{N}$ and $a$ is obtained from JKR fitting. It can be concluded from the comparisons between (a) and (c) that the activation energy increases rapidly as the contact size increases. 


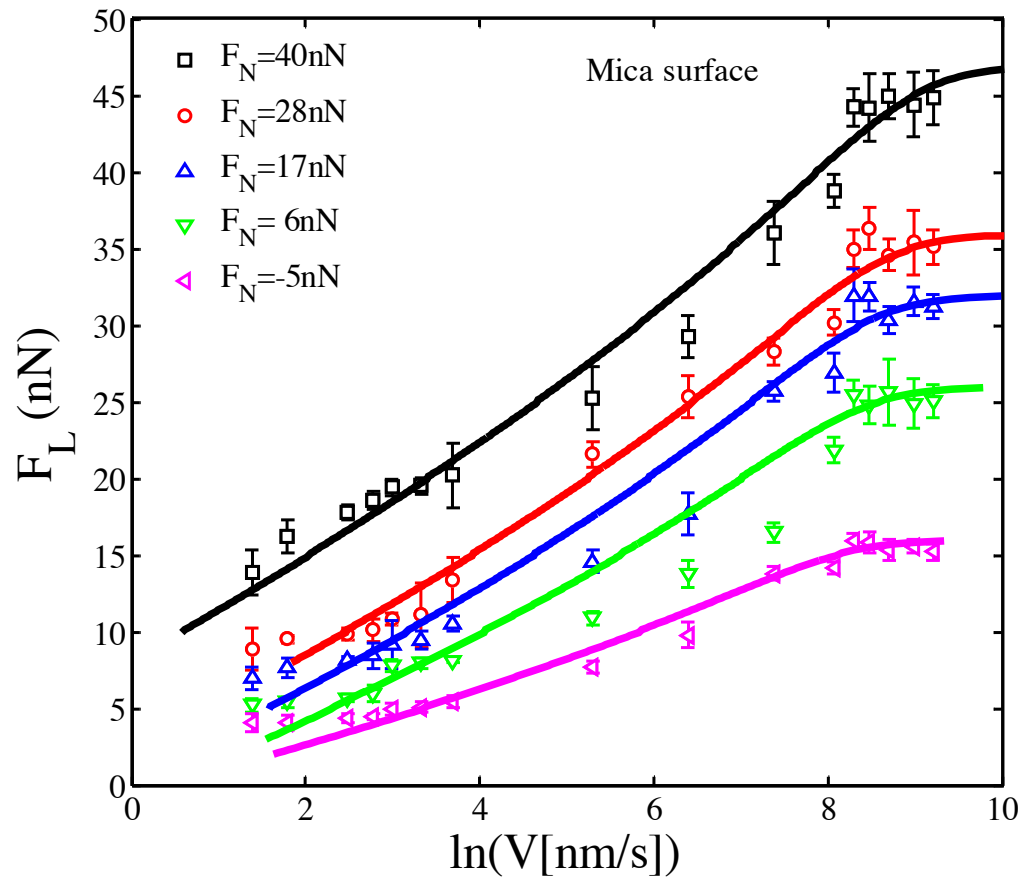

(a)

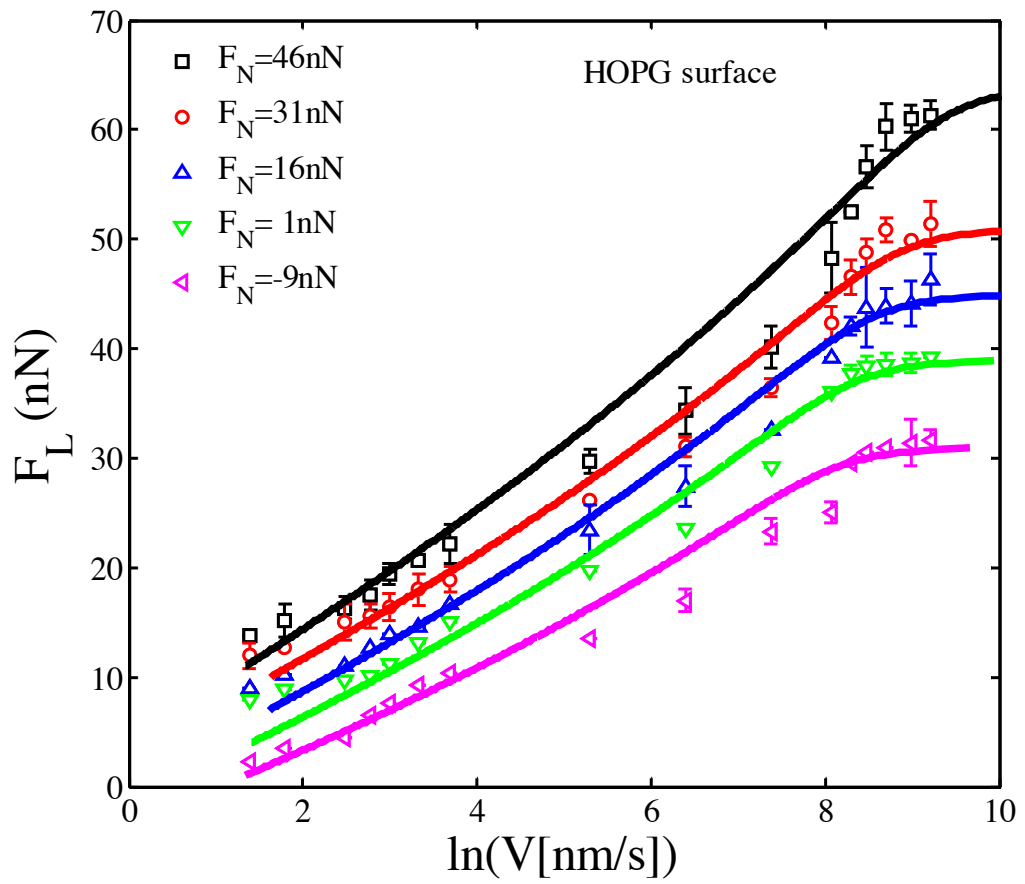

Figure 1 


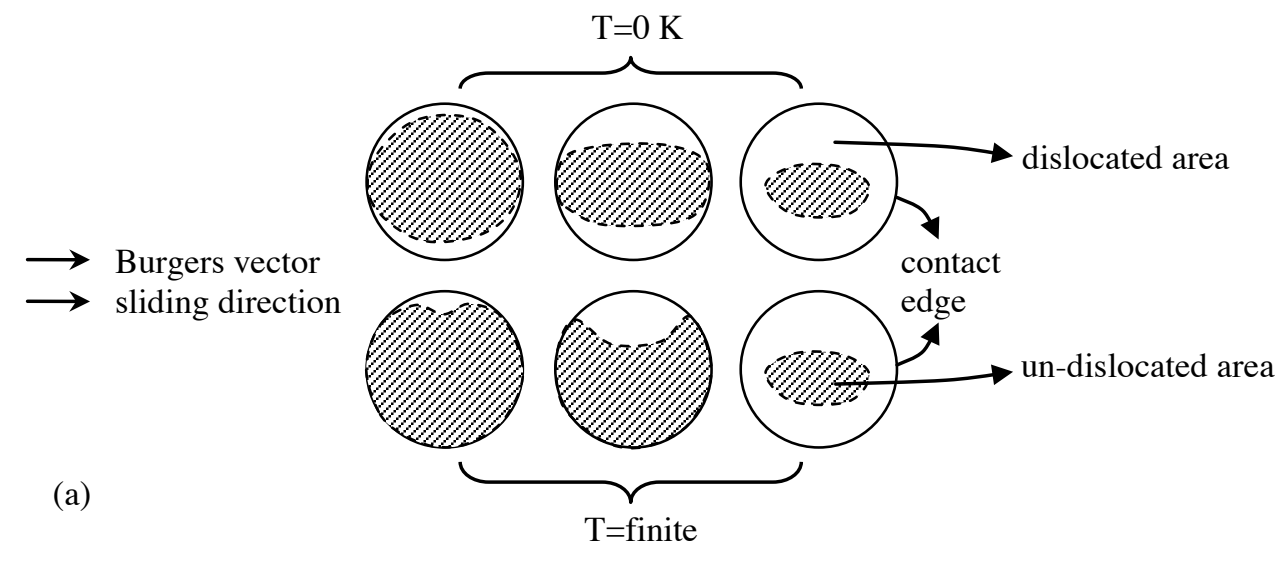

(b)
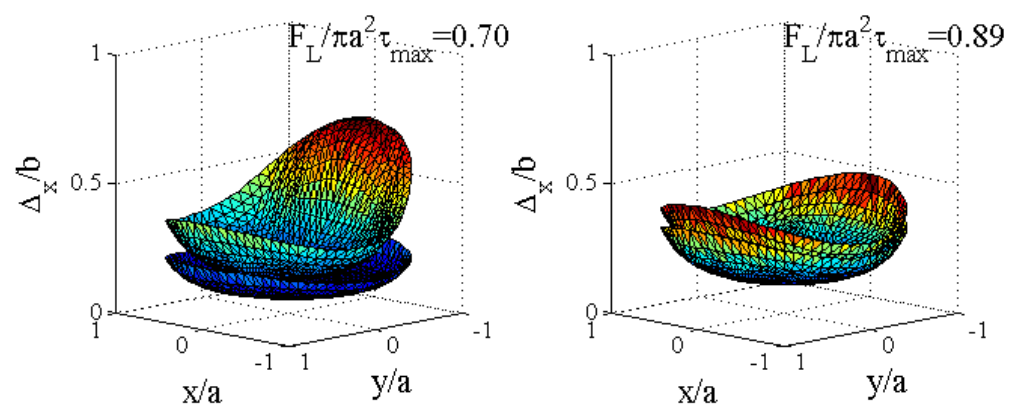

(c)

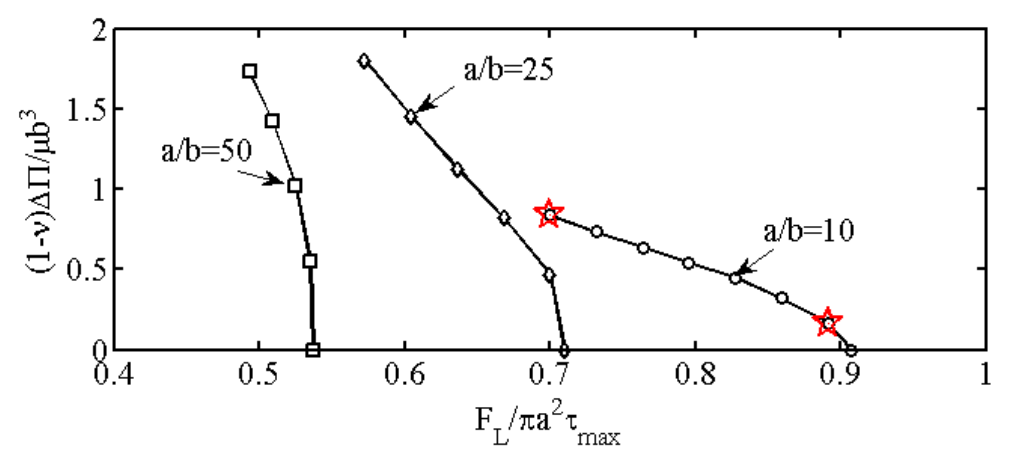

Figure 2 
(a)

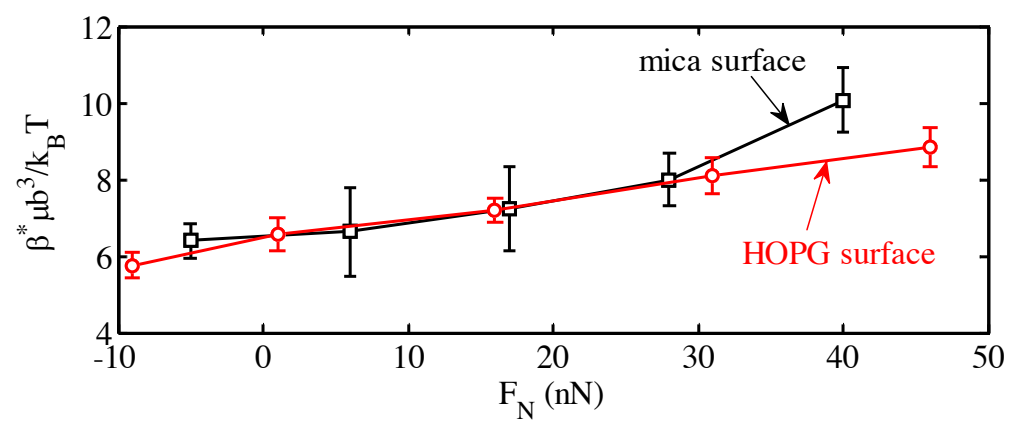

(b)

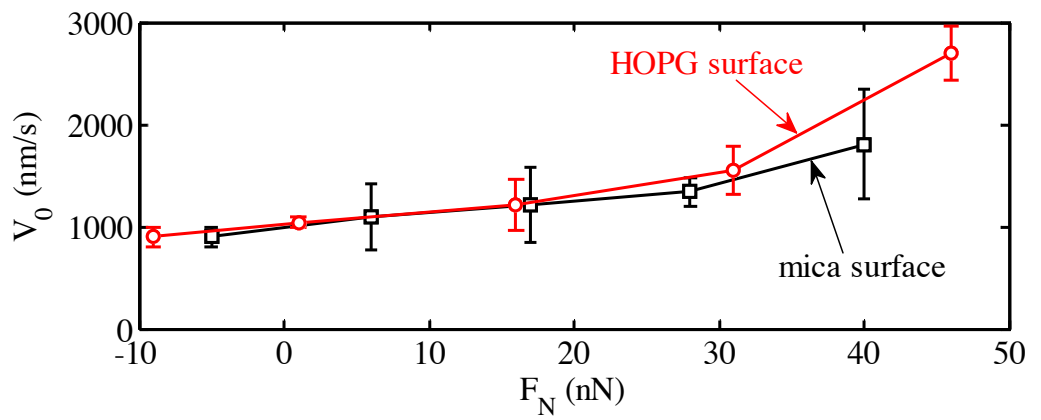

(c)

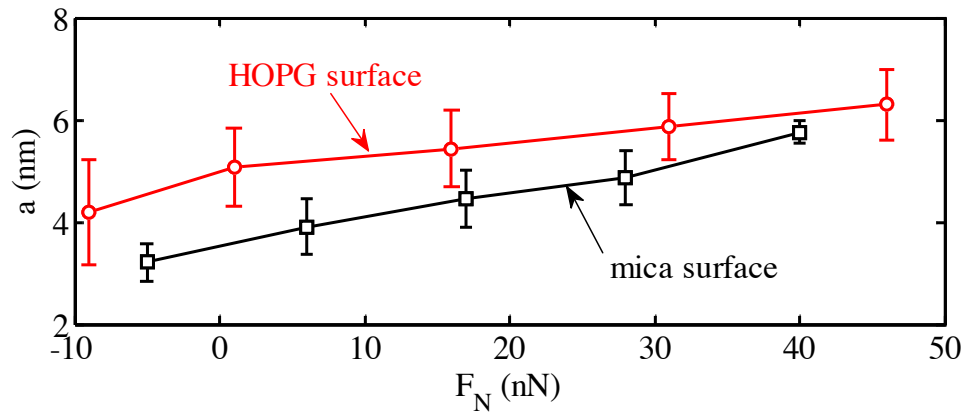

Figure 3 